\title{
Prognostic importance of myocardial injury in critically ill dogs with systemic
} inflammation

Langhorn, Rebecca; Oyama, M. A.; King, L. G.; Machen, M. C.; Trafny, D. J.; Thawley, V.; Willesen, Jakob; Tarnow, Inge; Kjelgaard-Hansen, Mads

Published in:

Journal of Veterinary Internal Medicine

DOI:

10.1111/jvim. 12105

Publication date:

2013

Document version

Early version, also known as pre-print

Citation for published version (APA):

Langhorn, R., Oyama, M. A., King, L. G., Machen, M. C., Trafny, D. J., Thawley, V., Willesen, J., Tarnow, I., \& Kjelgaard-Hansen, M. (2013). Prognostic importance of myocardial injury in critically ill dogs with systemic inflammation. Journal of Veterinary Internal Medicine, 27(4), 895-903. https://doi.org/10.1111/jvim.12105 


\title{
Prognostic Importance of Myocardial Injury in Critically Ill Dogs with Systemic Inflammation
}

\author{
R. Langhorn, M.A. Oyama, L.G. King, M.C. Machen, D.J. Trafny, V. Thawley, J.L. Willesen, \\ I. Tarnow, and M. Kjelgaard-Hansen
}

\begin{abstract}
Background: In noncardiac critical disease in humans, myocardial injury as detected by cardiac troponin I and $\mathrm{T}(\mathrm{cTnI}$ and $\mathrm{cTnT}$ ) has been linked to high intensive care unit (ICU) death independent of prognostic composite scoring.

Hypothesis: Presence of myocardial injury predicts short-term death in critically ill dogs with systemic inflammation and provides additional prognostic information when combined with established canine prognostic composite scores.

Animals: Forty-two dogs admitted to the ICU with evidence of systemic inflammation and no primary cardiac disease.

Methods: Prospective cohort study. Blood samples were obtained at ICU admission for the measurement of cTnI and cTnT, C-reactive protein, and several cytokines. The acute patient physiologic and laboratory evaluation (APPLE) score and the survival prediction index were calculated within the first 24 hours of admission. Receiver operating characteristic (ROC) curves were used to examine the prognostic capacity of each biomarker and severity score. Multiple logistic regression analysis was performed to evaluate whether cardiac markers significantly contributed to severity scores.

Results: Twenty-eight day case fatality rate was $26 \%$ (11/42 dogs). cTnI concentrations were (median [range]) 0.416 [0.004-141.5] ng/mL and cTnT concentrations were $13.5[<13-3,744] \mathrm{ng} / \mathrm{L}$. cTnI, cTnT, and the APPLE score were all significant prognosticators with areas under the ROC curves [95\% CI] of 0.801 [0.649; 0.907], 0.790 [0.637; 0.900], and 0.776 $[0.621 ; 0.889]$, respectively. cTnI significantly contributed to the APPLE score in providing additional prognostic specificity $(P=.025)$.
\end{abstract}

Conclusions and Clinical Importance: Markers of myocardial injury predict short-term death in dogs with systemic inflammation and cTnI significantly contributes to the APPLE score.

Key words: Biomarker; Cardiac troponin; Companion animals; Severity scores.

C ritical illness can be defined as important metabolic derangements that require intensive care to sustain life or enhance metabolic stability. ${ }^{1}$ Critically ill patients have a high risk of death, and the ability to measure markers of deterioration is crucial as it could allow the possibility to intervene with additional or alternate treatment. Prognostic composite scores are continuously being examined and developed in human ${ }^{2}$ and veterinary medicine, with main components being markers of organ dysfunction. Two diagnosisindependent severity scores have been validated for use in dogs, the acute patient physiologic and laboratory evaluation (APPLE) ${ }^{3}$ and the survival prediction index (SPI2). ${ }^{4}$ These scores, however, do not contain any markers of myocardial injury, and contribution of

From the Department of Veterinary Clinical and Animal Sciences, University of Copenhagen, Frederksberg C, Denmark (Langhorn, Willesen, Kjelgaard-Hansen); the Department of Clinical Studies - Philadelphia, School of Veterinary Medicine, University of Pennsylvania, Philadelphia, PA (Oyama, King, Machen, Trafny, Thawley); and Chr. Hansen A/S, Horsholm, Denmark (Tarnow). This study was carried out at the Matthew J. Ryan Veterinary Hospital of the University of Pennsylvania, Philadelphia, PA, in summer 2011. Part of the study was presented at the 2012 American College of Veterinary Internal Medicine Forum, New Orleans, LA, May 31 to June 2, 2012 .

Corresponding author: R. Langhorn, Department of Veterinary Clinical and Animal Sciences, University of Copenhagen, Groennegaardsvej 3, ground floor, DK-1870 Frederiksberg C, Denmark; e-mail: rel@sund.ku.dk.

Submitted September 21, 2012; Revised February 19, 2013; Accepted April 8, 2013.

Copyright (C) 2013 by the American College of Veterinary Internal Medicine

$10.1111 /$ jvim. 12105

$\begin{array}{ll}\text { Abbreviations: } \\ \text { AMI } & \text { acute myocardial infarction } \\ \text { APACHE } & \text { acute physiology and chronic health evaluation } \\ \text { APPLE } & \begin{array}{l}\text { acute patient physiologic and laboratory evaluation } \\ \text { area under the curve }\end{array} \\ \text { AUC } & \text { cardiac troponin I } \\ \text { cTnI } & \text { cardiac troponin T } \\ \text { cTnT } & \text { intensive care unit } \\ \text { ICU } & \text { interleukin } \\ \text { IL } & \text { monocyte chemo-attractant protein-1 } \\ \text { MCP-1 } & \text { receiver operating characteristic } \\ \text { ROC } & \text { simplified acute physiology score } \\ \text { SAPS } & \text { survival prediction index } \\ \text { SPI2 } & \text { tumor necrosis factor- } \alpha \\ \text { TNF- } \alpha & \text { Veterinary Hospital of the University of Pennsylvania } \\ \text { VHUP } & \end{array}$

such injury to the prognostic capacity of severity scores in dogs is unexplored.

In humans, cardiac troponins $\mathrm{I}$ and $\mathrm{T}$ (cTnI and cTnT) have long been recognized as sensitive and specific markers of myocardial injury which are most commonly used in the diagnosis of acute myocardial infarction (AMI). ${ }^{5}$ However, there is an increasing awareness that myocardial injury occurs much more frequently than previously suspected in critically ill human patients with primary disease of noncardiac origin. $^{6-9}$ In the noncardiologic human ICU, sepsis is the most common cause of death. ${ }^{10}$ Myocardial injury in patients with sepsis or other causes of systemic inflammation is presumably caused by hemodynamic changes, toxic effects of cytokines, microthrombosis, and ischemia-reperfusion injury. ${ }^{7,11-14}$ This myocardial injury, though often clinically unrecognized, has been 
associated with increased morbidity and death, ${ }^{7-9,13,15}$ and detecting and treating it might be all the more important as it might be caused by cardiomyocyte leakage of the cytosolic pool of troponins rather than cell necrosis, thereby being potentially reversible. ${ }^{6,16}$ Interestingly, no established correlation between cardiac troponin concentrations and prognostic composite scores in humans, such as the acute physiology and chronic health evaluation (APACHE) II or III or the simplified acute physiology score (SAPS) II, has been found. ${ }^{7,12,13,17,18}$ As the significance of myocardial injury or dysfunction was not included when these models were developed, this had led to speculation that cardiac troponins can provide additional prognostic information, 7,13 and several human studies have concluded that cardiac troponin remains an independent prognosticator even after stratifying for degree of illness severity. ${ }^{13,17}$ In dogs, the established prognostic composite scores, APPLE and SPI2, also lack markers of myocardial injury.

The objective of this study was to investigate the presence and prognostic relevance of myocardial injury in critically ill dogs with systemic inflammation. It was hypothesized that the presence of myocardial injury would be predictive of short-term mortality in dogs admitted to the ICU with systemic inflammation, and that it would provide additional prognostic information when added to established prognostic composite scores in dogs. As the myocardial injury is thought to be mediated by inflammatory cytokines, ${ }^{6,14,16}$ a 2 nd objective of the study was to investigate the possible contribution of cytokines to prognostic composite models with an included marker of myocardial injury.

\section{Materials and Methods \\ Study Population}

Over a 3-month period from May to July 2011, critically ill client-owned dogs were included at the time of admission to the ICU at the Veterinary Hospital of the University of Pennsylvania (VHUP). The study protocol was approved by the VHUP Privately Owned Animal Protocol Committee and the University of Pennsylvania Institutional Animal Care and Use Committee, and informed consent was obtained from all owners. The study was performed as a prospective cohort study with 28 days of follow-up. Dogs that had been diagnosed with cardiac disease before admission were excluded. An echocardiographic examination was performed by a board-certified cardiologist or cardiology resident and a 5-min EKG recorded. Dogs in which evidence of structural primary heart disease (symptomatic or asymptomatic) was identified were further excluded. Likewise, dogs recently treated with a known cardiotoxic drug (eg, doxorubicin) were excluded. Finally, dogs were excluded if data collection posed a risk to the dog (ie, if the dog was too small for collection of the required blood volume, had severe respiratory distress, or severe anemia), if study participation was declined by the owner or attending clinician, or if they were euthanized for reasons other than a grave prognosis for survival (eg, financial reasons).

Systemic inflammation at ICU admission was defined as a serum concentration of C-reactive protein (CRP) $>35 \mathrm{mg} / \mathrm{L}$ within the first 24 hours of admission. Dogs with CRP concentrations below this cut-off were excluded from the study when results of the CRP analysis were available. Based on clinical, hematological, and biochemical findings, an SPI2 (a logistic regression formula based on the parameters PCV, creatinine, albumin, mean arterial pressure, respiratory rate, age, and admitting service [medicine or surgery] ${ }^{4}$ and an APPLE (a scoring system based on the parameters WBC, creatinine, total bilirubin, albumin, lactate, $\mathrm{Sp}_{2}$, respiratory rate, age, mentation, and presence of fluid in one or more body cavities) $\mathrm{score}^{3}$ were calculated for each dog with systemic inflammation. Both were calculated within 24 hours of ICU admission according to previously described methods. Clinical outcome was defined as survival or mortality 28 days postadmission and was determined through follow-up visits or telephone contact with the owner.

Healthy animals for a control group were recruited from hospital staff and veterinary students and determined healthy through physical examination, hematological and biochemical profiles, and echocardiography.

\section{Blood Sampling and Analyses}

For each dog, a hematological and biochemical analysis was performed at the time of ICU admission with automated analyzers, ${ }^{\text {b,c }}$ and cross checked by a board-certified clinical pathologist. Serum for analysis of cTnI, cTnT, and cytokines was obtained at the time of ICU admission and for CRP analysis at admission and 12-24 hours later. Serum was collected into gel separator tubes, allowed to clot for 30 minutes at room temperature, centrifuged for 10 minutes at $1,300 \times g$, separated, and stored in cryovials at $-70^{\circ} \mathrm{C}$ within 2 hours of blood collection. Samples were stored for a maximum of 8 months until batch analysis. cTnI and cTnT were analyzed by commercially available high-sensitivity immunoassays. ${ }^{\mathrm{d}, \mathrm{e}}$ The cTnI assay has recently been validated for use in companion animals, ${ }^{19}$ and the cTnT assay has been used previously for assessment of myocardial injury in dogs. ${ }^{20} \mathrm{CRP}$ was analyzed by a commercially available turbidimetric immunoassay validated for canine use ${ }^{21}$ and calibrated with purified canine CRP. ${ }^{\mathrm{f}}$ Serum cytokine activity was assessed by a canine-specific tumor necrosis factor- $\alpha$ (TNF- $\alpha$ ) assay $^{\mathrm{g}}$ (detection limit: $2.4 \mathrm{pg} / \mathrm{mL}$ ) and a canine-specific multiplex assay ${ }^{\mathrm{h}}$ for interleukin-10 (IL-10) (detection limit: $1.6 \mathrm{pg} / \mathrm{mL}$ ), IL-15 (detection limit: $14.8 \mathrm{pg} / \mathrm{mL}$ ), IL-18 (detection limit: $4.6 \mathrm{pg} / \mathrm{mL}$ ), and monocyte chemotactic protein-1 (MCP-1) (detection limit: $8.6 \mathrm{pg} / \mathrm{mL}$ ) with an automated analyzer. ${ }^{\mathrm{i}}$ The assays included internal quality control material.

\section{Statistical Analyses}

Data were assessed for normality by D'Agostino-Pearson omnibus test. Logarithmic transformation was applied where this assured a Gaussian distribution of otherwise nonparametric data. A two-tailed $t$-test was used to compare Gaussian data, and the Mann-Whitney $U$-test was used to compare non-Gaussian data. In a few cases, because of the fact that all control dogs had cTnT and IL-10 values below the detection limits of the assays, these animals were set to have concentrations corresponding to the detection limits, and the Wilcoxon Signed Rank test was used to test the data against a hypothetical value (the cut-off value of the assay in question). Correlations between cardiac markers, severity scores, and cytokines were assessed graphically as well as by Pearson's or Spearman's correlation coefficient where appropriate. The prognostic capability for 28-day mortality of the SPI2 and APPLE score models as well as of markers of cardiac injury (cTnI and $\mathrm{cTnT}$ ) were assessed by receiver operating characteristic (ROC) curve analysis. A significant prognosticator was defined as having an area under the curve (AUC) significantly 
greater than 0.5 . Where the AUC for a prognostic composite score and for a cardiac marker was significantly greater than 0.5 , the cardiac marker was added to the scoring model by multiple logistic regression to determine whether it provided additional prognostic information to the model. The optimal combination of composite score and cardiac marker was obtained in a forward inclusion fashion. ${ }^{22}$ To investigate the suggested mediation of myocardial injury by inflammatory cytokines and its relation to prognosis, ROC curves were also created for each cytokine investigated, and those that had AUCs significantly greater than 0.5 were then added into the model individually in a forward inclusion fashion. Troponin and cytokine concentrations were logarithmically transformed to achieve Gaussian distribution for multiple logistic regression. Statistical significance was defined as $P<.05$. All statistical analyses were conducted by commercial statistical software (Normality, $t$-test, Mann-Whitney $U$-test, Wilcoxon's test, correlations, ${ }^{\mathrm{j}}$ ROC curve analysis, ${ }^{\mathrm{k}}$ and multiple logistic regression ${ }^{1}$.

\section{Results}

\section{Study Population Characteristics}

One hundred and six dogs were admitted to the ICU during the study period. Of these, 39 were excluded because of cardiac disease. Twelve dogs were excluded because data collection posed a risk to the animal, and four were excluded because the owner or the attending clinician declined inclusion of the dogs. Two dogs died in the ICU before inclusion in the study. Of the remaining 49 dogs, six did not meet the criteria of systemic inflammation at the time of admission and were excluded. In addition, 1 dog was excluded because it was euthanized because of a grave prognosis for mobility rather than a grave prognosis for survival. Thus, the study population consisted of 42 dogs. The 42 dogs were 1 female intact, 20 female spayed, and 21 male castrated dogs with an age span of 1-14 years (mean 7.0 years). Eight dogs were mixed breeds; all other dogs were purebreds of 23 different breeds, the most frequent being Labrador Retriever $(n=6)$, Boxer $(n=3)$, and French Bulldog $(n=3)$. Dogs presented with a primary diagnosis of trauma $(\mathrm{n}=10,5$ dogs with polytrauma and 5 dogs with localized trauma), neoplasia $(\mathrm{n}=8)$, gastrointestinal disease $(n=8)$, respiratory disease $(n=5)$, neurological disease $(n=4)$, hematological disease $(n=2)$, and various $(\mathrm{n}=5$ : hemoabdomen caused by rupture of splenic hematoma $[\mathrm{n}=2]$ and 1 of each of biliary mucocele, peritonitis, and GDV) diseases.

Eight dogs were used as healthy controls. The 8 dogs were 4 female spayed, 2 male intact, and 2 male castrated dogs with an age span of 1-9 years (mean 3.9 years). Two dogs were mixed breeds; all other dogs were purebreds of 6 different breeds.

\section{Clinical Outcome}

The 28-day case fatality rate was $26 \%$ (11/42 dogs). Ten dogs were euthanized during hospitalization because of the severity of clinical signs and perceived poor prognosis, and 1 dog died at home after discharge. One dog was lost to follow-up and was last seen 21 days after admission. The owner could not be reached after this date. As the dog was doing well on day 21 , it was considered a survivor.

\section{Severity Scores, Cardiac Troponins, and Cytokines}

The median [range] APPLE score of the 42 dogs with systemic inflammation was 35 [16-54]. Nonsurvivors (41 [16-54]) had significantly higher APPLE scores than survivors (31 [17-48]) (Mann-Whitney, $P=.0078$ ). The median [range] SPI2 score was 0.71 $[0.25-0.91]$. There was no significant difference between SPI2 scores for survivors $(0.72$ [0.33-0.9]) and nonsurvivors (0.58 [0.25-0.91]) (Mann-Whitney, $P=.11$ ).

Admission cTnI concentrations were (median [range]) $0.416[0.004-141.5] \mathrm{ng} / \mathrm{mL}$. Forty of the 42 dogs $(95.2 \%)$, including all nonsurvivors, had cTnI concentrations above the concentration range of the control dogs $(0.01[0.004-0.04] \mathrm{ng} / \mathrm{mL})$ at the time of ICU admission. Nonsurvivors (2.64 [0.24-85.17] $\mathrm{ng} / \mathrm{mL}$ ) had significantly higher cTnI concentrations than survivors $(0.20$ [0.004-141.5] $\mathrm{ng} / \mathrm{mL})$ (based on ROC analysis) and control dogs (0.01 [0.004-0.04] $\mathrm{ng} / \mathrm{mL})(t$-test, $P<.001)$, and survivors had significantly higher cTnI concentrations than control dogs $(t$-test, $P<.001)($ Fig 1A). Admission cTnT concentrations were $13.5[<13-3,744] \mathrm{ng} / \mathrm{L}$. Twenty-one dogs $(50 \%)$, including 10 of $11(91 \%)$ nonsurvivors, had cTnT concentrations above the detection limit of the assay $(13 \mathrm{ng} / \mathrm{L})$. All healthy dogs had cTnT concentrations below the detection limit. Nonsurvivors $(46[<13-$ 686] $\mathrm{ng} / \mathrm{L}$ ) had significantly higher cTnT concentrations than survivors $(<13[<13-3,744] \mathrm{ng} / \mathrm{L}$ ) (based on ROC analysis) and control dogs (all $<13 \mathrm{ng} / \mathrm{L}$ ) (Wilcoxon Signed Rank test, $P=.002$ ), and survivors had significantly higher $\mathrm{cTnT}$ concentrations than control dogs (Wilcoxon Signed Rank test, $P=.001$ ) (Fig 1B). Admission cTnI and cTnT concentrations were highly correlated $(r=0.91$, Spearman, $P<.001)$. No correlation was found between the cardiac troponins and the APPLE score $(r=0.20 \quad$ [Spearman, $P=.21]$ and $r=0.28$ [Spearman, $P=.07]$ for correlation with $\mathrm{cTnI}$ and cTnT, respectively) or the SPI2 $(r=-0.11$ [Spearman, $P=.51$ ] and $r=-0.15$ [Spearman, $P=.35$ ] for correlation with $\mathrm{cTnI}$ and $\mathrm{cTnT}$, respectively).

The APPLE score and both cardiac troponins were found to be significant prognosticators (Table 1). However, no significant prognostic capacity was identified for SPI2 in the examined cohort (Table 1). It was, therefore, not possible to investigate the significance of adding a marker of myocardial injury to this model.

The possible contribution of cardiac markers to the APPLE score was examined by multiple logistic regression. cTnI contributed significantly $(P=.025)$ in a model combining the APPLE score and cTnI (coefficient estimate $[95 \% \mathrm{CI}]: 1.01[0.04 ; 1.99])$ corresponding to an odds ratio of 28-day mortality of 2.7 for each 10 -fold increase in cTnI. There was no significant contribution of cTnT to the APPLE score $(P=.16)$. Graphing the $\mathrm{cTnI}$ concentration against the APPLE score revealed that both prognosticators 


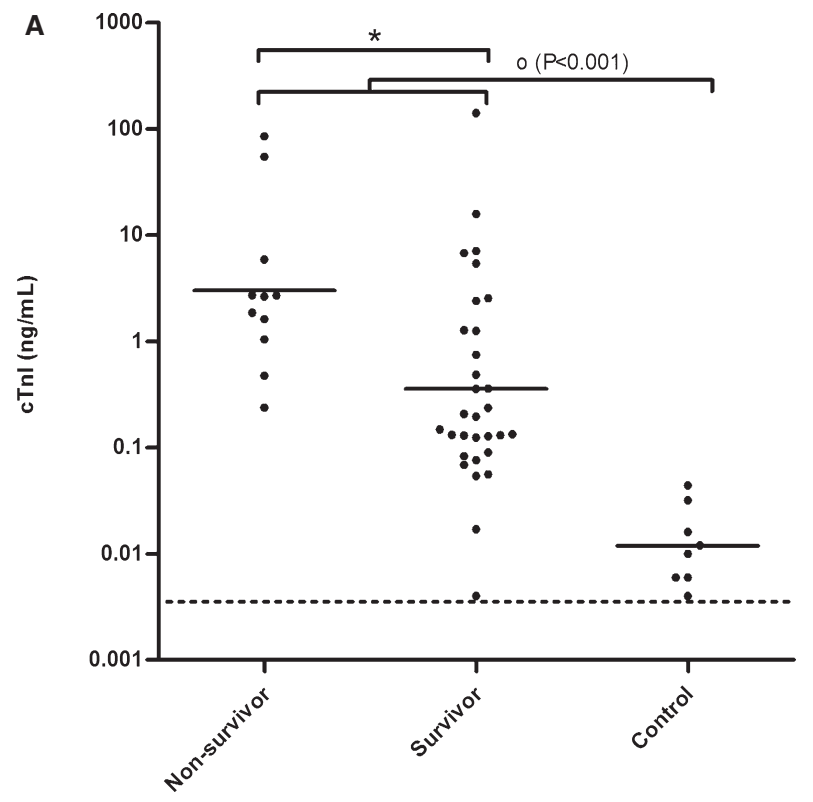

Table 1. Prognostic capacity of the established canine prognostic composite scores and cardiac troponins in 42 critically ill dogs with systemic inflammation evaluated by the analysis of receiver operating characteristic curves (ROCs).

\begin{tabular}{llcc}
\hline & Variable & AUC-ROC & 95\% CI of AUC-ROC \\
\hline Severity score & APPLE* & 0.776 & {$[0.621 ; 0.889]$} \\
& SPI2 & 0.610 & {$[0.447 ; 0.756]$} \\
Cardiac marker & cTnI* & 0.801 & {$[0.649 ; 0.907]$} \\
& cTnT* $^{*}$ & 0.790 & {$[0.637 ; 0.900]$} \\
\hline
\end{tabular}

AUC-ROC, area under the receiver operating characteristic curve; CI, confidence interval; APPLE, acute patient physiologic and laboratory evaluation; SPI2, survival prediction index; cTnI, cardiac troponin I; cTnT, cardiac troponin $\mathrm{T}$.

*Indicates significant prognostic capacity.
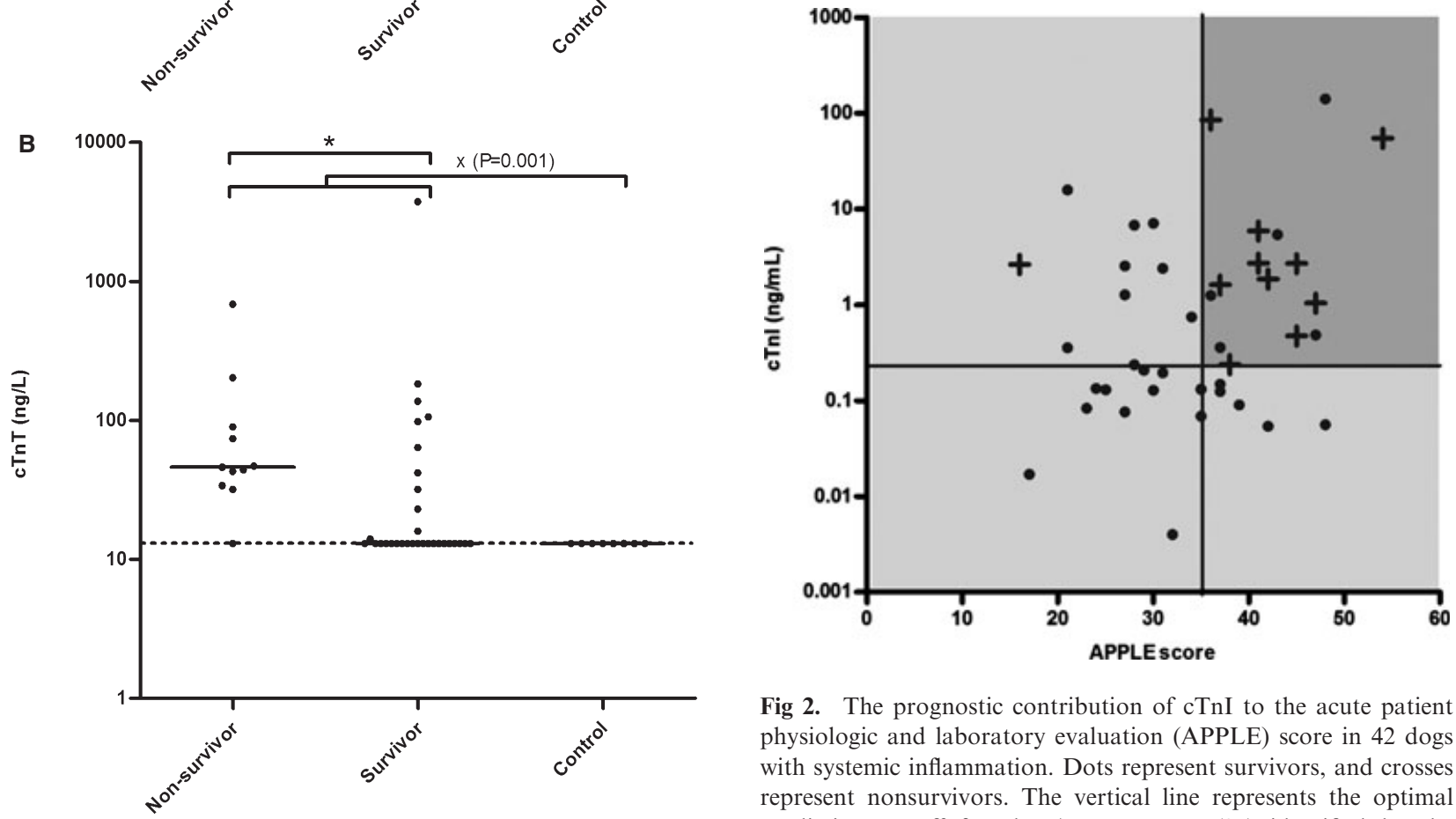

Fig 2. The prognostic contribution of cTnI to the acute patient physiologic and laboratory evaluation (APPLE) score in 42 dogs with systemic inflammation. Dots represent survivors, and crosses represent nonsurvivors. The vertical line represents the optimal predictive cut-off for the APPLE score (35) identified by the receiver operating characteristic (ROC) analysis. Dogs to the left of this line were predicted to survive by the APPLE score, and those to the right were predicted to die. The horizontal line represents the optimal predictive cut-off for cTnI $(0.24 \mathrm{ng} / \mathrm{mL})$ identified by the ROC analysis. Dogs below this line were predicted to survive by cTnI, and those above were predicted to die. The dark gray zone represents dogs predicted to die according to an agreed prediction of mortality of APPLE and cTnI. The light gray zone represents dogs predicted to survive by either APPLE or $\mathrm{cTnI}$.

had an excellent negative predictive value attributable to high prognostic sensitivity, but a less optimal positive predictive value attributable to compromised prognostic specificity (many false positives) when applied alone. The combination of cTnI and APPLE clearly reduced the number of false positives, and cTnI thus seemed to improve the prognostic specificity (and thereby the positive predictive value) of the model (Fig 2). As all animals that died had cTnI

values above the optimal predictive cut-off $(0.24$ $\mathrm{ng} / \mathrm{mL}$ ) identified by the ROC analysis, the number of true positives (nonsurvivors predicted to die) - and thereby the prognostic sensitivity of the APPLE scorewas not compromised by the inclusion of cTnI. For animals with cTnI concentrations above $0.24 \mathrm{ng} / \mathrm{mL}$, 
the mortality rate was $45.8 \%$ whereas it was $0 \%$ for animals with concentrations below the cut-off.

One measurement of TNF- $\alpha$ was missing because of insufficient sample material. For TNF- $\alpha$ and MCP-1 there was a significant difference between dogs with systemic inflammation (TNF- $\alpha$ : $10.10 \quad$ [6.36-23.35] $\mathrm{pg} / \mathrm{mL}$; MCP-1: 656.4 [104.6-1,609] pg/mL) and control dogs (TNF- $\alpha$ : 8.17 [6.26-9.42] pg/mL; MCP-1: 110.2 [94.69-198.9] $\mathrm{pg} / \mathrm{mL}$ ), however, no difference was found between nonsurvivors (TNF- $\alpha$ : 10.52 [7.5718.41] pg/mL; MCP-1: 743.7 [193.6-1,506] pg/mL) and survivors (TNF- $\alpha$ : 10.1 [6.36-23.35] pg/mL; MCP-1: $591.8[104.6-1,609] \mathrm{pg} / \mathrm{mL}$ ) (Fig 3D,E). For IL-15 and IL-18 there was no significant difference between dogs with systemic inflammation (IL-15: $28.73[<14.8-1,738]$ pg/mL; IL-18: $69.63[<4.6-1,689] \mathrm{pg} / \mathrm{mL})$ and control dogs (IL-15: 74.12 [24-307.5] pg/mL; IL-18: 89.22 [4.6-658] pg/mL), however, a significant difference was found between nonsurvivors (IL-15: 16 [ $<14.8-457.2]$ $\mathrm{pg} / \mathrm{mL}$; IL-18: $26.71[<4.6-293.5] \mathrm{pg} / \mathrm{mL}$ ) and survivors (IL-15: $60.23[<14.8-1,738] \mathrm{pg} / \mathrm{mL}$; IL-18: $79.39[<4.6-$ $1,689] \mathrm{pg} / \mathrm{mL}$ ) (Fig 3B,C). For IL-10 there was a significant difference between dogs with systemic inflammation $(8.5[<1.6-145.6] \mathrm{pg} / \mathrm{mL})$ and control dogs (all $<1.6 \mathrm{pg} / \mathrm{mL}$ ) as well as between nonsurvivors $(14.33[<1.6-98.64] \mathrm{pg} / \mathrm{mL})$ and survivors $(7.05[<1.6-$ 145.6] $\mathrm{pg} / \mathrm{mL}$ ) (Fig 3A). A significant correlation was found between cTnI and the cytokines IL-15 $(r=-0.48$, Spearman, $P=.012)$ and IL-10 $(r=0.46$, Pearson, $P=.0023$ ), but no correlation was found with TNF- $\alpha(r=0.06$, Spearman, $P=.71)$, IL-18 $(r=-0.16$, Pearson, $P=.32)$, or MCP-1 $(r=-0.06$, Spearman, $P=.71)$.

IL-10, IL-15, and IL-18 were found to be significant prognosticators whereas TNF- $\alpha$ and MCP-1 were not (Table 2). Only IL-15 contributed significantly to the combined prognostic model of cTnI and APPLE $(P=.024)$, and $\mathrm{cTnI}$ lost its significance when IL-15 was added to the model $(P=.16)$.

\section{Discussion}

This study documents a clinically important degree of myocardial cell injury occurring in dogs with systemic inflammation and without primary cardiac disease. Presence of myocardial injury was predictive of short-term survival, and cTnI as a marker of myocardial injury contributed independently to the established prognostic composite score, APPLE, by providing additional prognostic specificity without compromising prognostic sensitivity. The inflammatory cytokine IL-15 was correlated with cTnI and identified as a significant coexplanatory factor for the prognostic value of $\mathrm{cTnI}$.

The importance of the heart in critical disease is hardly surprising. However, although objective markers of injury and function for several other organs have been routinely measured for decades, no cardiac marker has been a part of a routine work-up in critically ill dogs. It seems from the findings of this study that the cardiac troponins, especially cTnI, may be useful for this purpose. The superiority of cTnI over cTnT in this study may be because of the fact that, although they supply similar information, cTnI levels generally increase with less severe disease than those of $\mathrm{cTnT}$, suggesting that $\mathrm{cTnI}$ is a more sensitive marker of myocardial injury. ${ }^{23,24}$ The reason for this difference in release is unknown, but may be because of a difference in protein size and molecular weight or the fact that cTnI may be degraded into fragments after the brief periods of myocardial ischemia. ${ }^{25,26}$ In cases of irreversible myocardial injury, it has also been speculated that cTnT may have a structurally closer binding to the tropomyosin chain than $\mathrm{cTnI} .{ }^{24}$

Human studies have revealed that the presence of myocardial injury is associated with increased morbidity and mortality, though clinically it is often unrecognized. ${ }^{8,9}$ In fact, patients with increased troponin concentrations can have similar clinical characteristics as those without evidence of cardiac injury. ${ }^{9}$ Considering that cardiac troponins are primarily used in the diagnosis of AMI, it is notable that mortality for critically ill patients with myocardial injury can be as high as or higher than that of patients in whom cardiac troponins were increased because of a primary cardiac disease or AMI. ${ }^{8,15,27}$ This is even more interesting when it is considered that patients with clinically unrecognized cardiac injury generally have significantly lower concentrations of cTnI than those with diagnosed AMI. ${ }^{8,28}$ Consequently, it seems that the presence of myocardial injury in itself rather than just the degree of injury accounts for a worse prognosis.

In several human studies, cTnI in critically ill patients remained an independent prognosticator even after stratifying for illness severity by use of a severity score. ${ }^{13,17}$ As is the case for veterinary severity scores, human severity scores such as APACHE and SAPS evaluate hemodynamic changes rather than the status of the heart itself, and it may be argued that myocardial injury, thus not accounted for by the scores, supplies additional prognostic information..$^{7,13}$ The contribution to the prognostic capacity of the APPLE score by cTnI in this study was highly significant. The effect of $\mathrm{cTnI}$ was to improve the prognostic specificity of the model. As the model's prognostic sensitivity was not compromised, it may be of value to include a marker of myocardial injury in prognostic severity scores in the future. It is also noteworthy that the dogs included in this study had received varying degrees of volume resuscitation before ICU admission. The prognostic capacity of the cardiac markers was verified in spite of this possible interference, thereby further strengthening their use in a clinical setting.

Although the APPLE score in itself was a significant prognosticator in this study, surprisingly, SPI2 was not. The cohort of dogs in this study had a survival rate of $73.8 \%$ which is within the range of survival rates for which the SPI2 was validated. ${ }^{4}$ However, this cohort was selected based on the presence of systemic inflammation, and all dogs without evidence of systemic inflammation were excluded. As dogs with systemic inflammation were included in the construction 

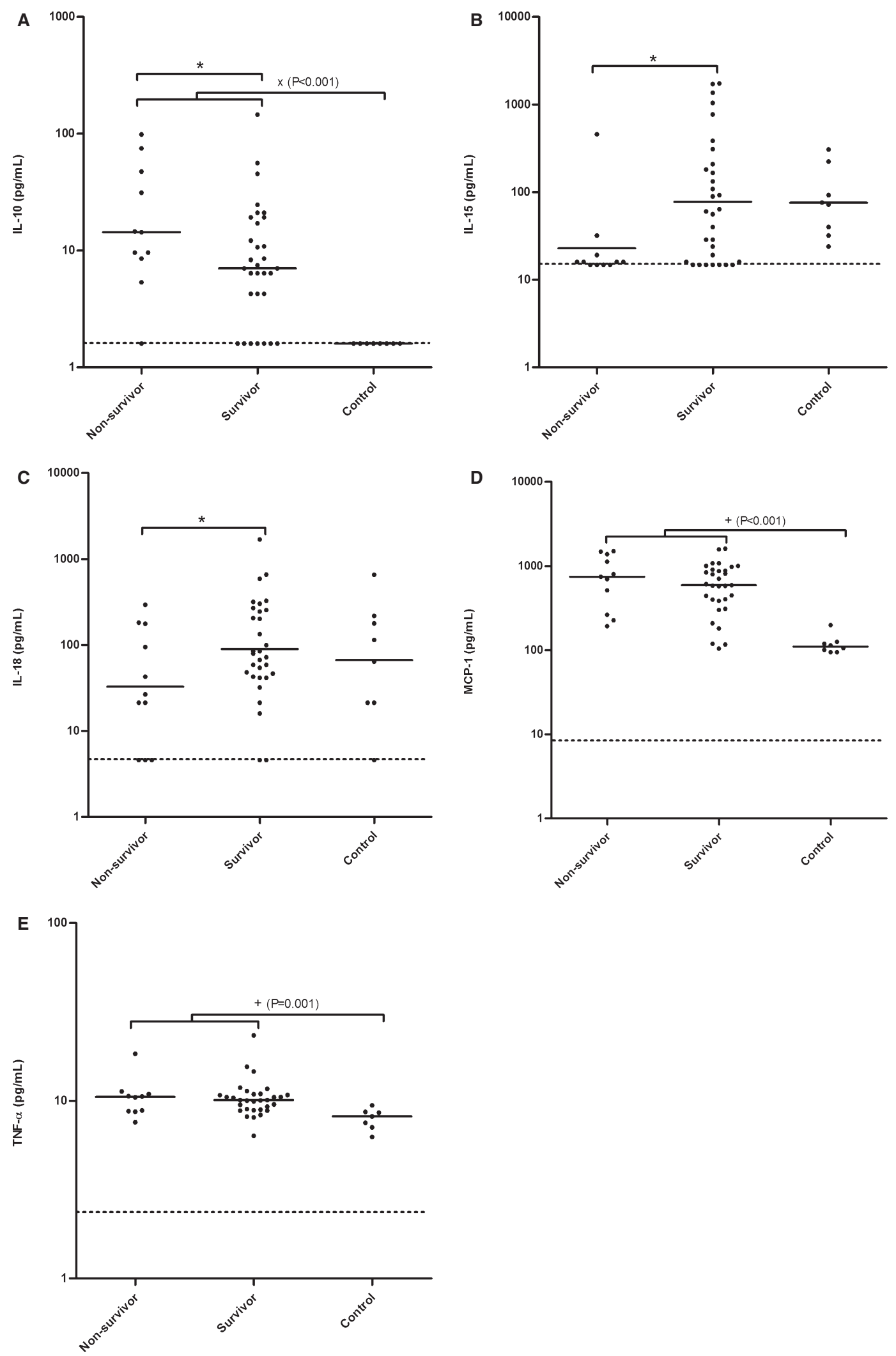

Fig 3. Serum interleukin (IL)-10 (A), IL-15 (B), IL-18 (C), monocyte chemo-attractant protein-1(MCP-1) (D), and tumor necrosis factor- $\alpha($ TNF- $\alpha)(\mathbf{E})$ concentrations of critically ill dogs with systemic inflammation $(n=42)$ and healthy control dogs $(n=8)$ as well as of survivors and non-survivors. Geometric mean of concentrations (B, C) for Gaussian and medians (A, D, E) for non-Gaussian data are shown as horizontal lines. Significant differences between groups are symbolized with *(receiver operating characteristic curve analysis with a 95\% confidence interval not including .5), $\mathrm{x}$ (Wilcoxon Signed Rank test), and + (Mann-Whitney $U$-test). 
Table 2. Prognostic capacity of various cytokines in 42 critically ill dogs with systemic inflammation evaluated by analysis of receiver operating characteristic curves (ROCs).

\begin{tabular}{llcc}
\hline & Variable & AUC-ROC & $95 \%$ CI of AUC-ROC \\
\hline Cytokine & IL-10* & 0.679 & {$[0.517 ; 0.814]$} \\
& IL-15* & 0.723 & {$[0.563 ; 0.849]$} \\
& IL-18* & 0.694 & {$[0.532 ; 0.826]$} \\
& MCP-1 & 0.575 & {$[0.413 ; 0.726]$} \\
TNF- $\alpha$ & 0.503 & {$[0.343 ; 0.663]$} \\
\hline
\end{tabular}

AUC-ROC, area under the receiver operating characteristic curve; CI, confidence interval; IL, interleukin; MCP-1, monocyte chemo-attractant protein- 1 ; TNF- $\alpha$, tumor necrosis factor- $\alpha$.

*Indicates significant prognostic capacity.

of both the SPI2 and the APPLE score, ${ }^{3,4}$ we used the scores in a patient group for which the scores have already been validated. Nevertheless, both scores were constructed with a less selected ICU population, giving a possible explanation for the reduced performance of the scores in these cohort when compared to construction and validation cohorts.

Prognostic composite scores assist clinicians in supplementing their clinical judgment with objective measures of patient illness and have been shown to improve clinicians' accuracy of survival prediction. ${ }^{29,30}$ Optimizing the predictive sensitivity and specificity of such scores is therefore desirable, and this study identifies a possible need for inclusion of the status of the myocardium in prognostic scoring. cTnI in itself as well as the APPLE score had an excellent negative predictive value, but a less optimal positive predictive value. This may be because of the fact that myocardial injury might be reversible in inflammatory diseases. Reversible myocardial depression associated with sepsis has been recognized in $40-50 \%$ of people with this disease, ${ }^{31,32}$ and in fact many of these people survive. ${ }^{33}$ Reversible myocardial injury has been explained by several theories. One theory advocates cytokinemediated myocardial cell injury leading to myocardial "cell membrane gaps" with leakage of troponins into the extracellular fluid. ${ }^{6,14,16}$ Another theory suggests reversible ischemia induced by an activated clotting system in sepsis or SIRS causing microcirculatory thrombosis, a theory that was rejected in a recent study. ${ }^{34}$ Finally, it has been postulated that reversible myocardial injury may be because of incomplete apoptosis. $^{35,36}$ Most likely, it is a combination of several factors. The theory of cytokine-mediated myocardial injury has gained most of the attention in research. Experimental studies indicate that cytokines such as TNF- $\alpha$ may lead to an increased permeability of the cardiomyocyte membrane for macromolecules such as the troponins. ${ }^{37,38}$ Cytokines are also thought to mediate decreased cardiac contractility ${ }^{39}$ which often accompanies sepsis. In 1 study of experimental endotoxemia, however, increased TNF- $\alpha$, IL-6, and IL-8 were not followed by an increase in cTnI, and it was speculated that a longer proinflammatory mediator exposure or a concurrent exposure to changes in additional cytokine concentrations were necessary for cardiac injury to take place. ${ }^{14}$ In this study, TNF- $\alpha$ was not correlated with cTnI or cTnT and was not a significant prognosticator in itself. The cytokines IL-10, IL-15, and IL-18, however, were significant prognosticators. IL-15 remained significant in a model combining the APPLE score and cTnI, and, interestingly, cTnI lost its significance when IL-15 was added to the model. This coexplanatory correlation as a predictor of outcome may be coupled to etiology and supports the suggested contribution of cytokines to myocardial injury. Experimental evidence exists of IL-15 treatment having a beneficial effect in mice with virus-induced myocarditis ${ }^{40}$ which further supports our findings of significantly higher cTnI concentrations and lower IL-15 concentrations in nonsurvivors compared to survivors. Further studies will have to be conducted to elucidate the possible etiological correlation.

An important limitation of this study was the fact that the model created combining the APPLE score with cTnI was not validated in a separate cohort of dogs. A multicenter study including a larger cohort of dogs with systemic inflammation would be valuable for further evaluation of the benefit of including markers of myocardial injury in composite severity scores. Secondly, a source of error inherent to veterinary survival studies was created when decisions were made to include dogs that were euthanized rather than only dogs that died naturally. Some nonsurvivors might, in fact, have survived, had treatment been continued. APPLE and SPI2, the 2 diagnosis-independent severity scores validated for use in dogs, both include euthanized dogs, but SPI2 attempts to minimize this error by only including dogs that are euthanized because of a grave prognosis for survival. ${ }^{3,4}$ Accordingly, these criteria were applied to this study as well. Thirdly, it cannot be excluded that a degree of mechanical cardiac injury may have occurred in the 5 dogs with polytrauma, causing potential bias. The dogs were equally distributed among the population with regard to cTnI concentrations and APPLE scores, exclusion from statistical analysis did not change any of the specific findings (data not shown), and as inflammatory cardiac injury was likely to be involved, these dogs were not excluded from the study. A 4th limitation was the fact that the analytical sensitivity of the assays for cTnT and IL-10 was insufficient, where the value of the detection limit was assigned to several samples with a risk of violation of statistical assumptions. However, as graphical examination of the data for both variables revealed a considerable difference between dogs with systemic inflammation and control dogs, the test results were considered valid. Finally, to ensure exclusion of primary cardiac disease, it is possible that a few dogs with decreased contractility were excluded as it may resemble mild DCM. This may have reduced the size of our study population, but was not thought to cause a bias as it only strengthened our certainty of a cardiac disease-free population. Nevertheless, a few study-eligible dogs with critical 
illness-induced myocardial dysfunction may have been wrongly excluded, but valvular disease was, by far, the most common cause of exclusion.

In conclusion, markers of myocardial injury were found to be predictive of 28-day case fatality in critically ill dogs with systemic inflammation and without a primary cardiac diagnosis. cTnI significantly contributed to the prognostic composite score, APPLE, by improving prognostic specificity without compromising prognostic sensitivity and thus may be valuable in construction of future severity scores. Finally, IL-15 might play a role in the sequence of events leading to myocardial injury in these dogs.

\section{Footnotes}

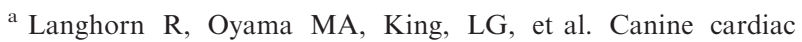
troponin I significantly complements established prognostic composite score in dogs with systemic inflammation. J Vet Intern Med 2012;26:713 (abstract)

${ }^{\mathrm{b}}$ Scil vet $\mathrm{ABC}$ hematology analyzer; Horiba ABX, Northampton, UK

${ }^{c}$ Vitros 350 chemistry analyzer; Orthoclinial Diagnostics, Johnson and Johnson, Rochester, NY

${ }^{d}$ ADVIA Centaur CP TnI-ultra; Siemens Healthcare Diagnostics Inc, Tarrytown, NY

e Elecsys hs-TnT; Roche Diagnostic Corporation, Indianapolis, IN

${ }^{\mathrm{f}}$ Canine C-reactive Protein; LifeDiagnostics, West Chester, PA

${ }^{g}$ Quantikine CATA00; R\&D Systems Inc, Minneapolis, MN

${ }^{\text {h }}$ CCYTO-90K; Millipore Corporation, Billerica, MA

${ }^{i}$ Luminex 200; Luminex Corporation, Austin, TX

${ }^{j}$ GraphPad Prism 5.01 for Windows; GraphPad Software, San Diego, CA

${ }^{\mathrm{k}}$ MedCalc 6.00.012; MedCalc Software, Mariakerke, Belgium

${ }^{1}$ SAS 9.2; SAS Institute Inc, Cary, NC

\section{Acknowledgments}

The authors thank Prof. Jens Peter Goetze, Department of Clinical Biochemistry, Rigshospitalet, University of Copenhagen, Denmark, for assistance with cardiac troponin $\mathrm{T}$ measurements and the staff at Matthew J. Ryan Veterinary Hospital of the University of Pennsylvania for help with inclusion of dogs for the study.

Part of the study was funded through research grants from Knud Højgaard's Fund, Denmark, and the Oticon Fund, Denmark.

Conflict of Interest Declaration: Authors disclose no conflict of interest.

\section{References}

1. Nelson OL, Thompson PA. Cardiovascular dysfunction in dogs associated with critical illnesses. J Am Anim Hosp Assoc 2006;42:344-349.

2. Le Gall J. The use of severity scores in the intensive care unit. Intensive Care Med 2005;31:1618-1623.
3. Hayes G, Mathews K, Doig G, et al. The acute patient physiologic and laboratory evaluation (APPLE) score: A severity of illness stratification system for hospitalized dogs. J Vet Intern Med 2010;24:1034-1047.

4. King LG, Wohl JS, Manning AM, et al. Evaluation of the survival prediction index as a model of risk stratification for clinical research in dogs admitted to intensive care units at four locations. Am J Vet Res 2001;62:948-954.

5. Thygesen K, Alpert JS, White HD. Universal definition of myocardial infarction. J Am Coll Cardiol 2007;50:2173-2195.

6. Ammann P, Fehr T, Minder EI, et al. Elevation of troponin I in sepsis and septic shock. Intensive Care Med 2001;27: 965-969.

7. Ammann P, Maggiorini M, Bertel O, et al. Troponin as a risk factor for mortality in critically ill patients without acute coronary syndromes. J Am Coll Cardiol 2003;41:2004-2009.

8. Guest T, Ramanathan A, Tuteur P, et al. Myocardial injury in critically ill patients-A frequently unrecognized complication. J Am Med Assoc 1995;273:1945-1949.

9. Wright RS, Williams BA, Cramner H, et al. Elevations of cardiac troponin I are associated with increased short-term mortality in noncardiac critically ill emergency department patients. Am J Cardiol 2002;90:634-636.

10. Martin GS, Mannino DM, Eaton S, Moss M. The epidemiology of sepsis in the United States from 1979 through 2000. N Engl J Med 2003;348:1546-1554.

11. Noble JS, Reid AM, Jordan LVM, et al. Troponin I and myocardial injury in the ICU. Br J Anaesth 1999;82:41-46.

12. ver Elst KM, Spapen HD, Nguyen DN, et al. Cardiac troponins I and $\mathrm{T}$ are biological markers of left ventricular dysfunction in septic shock. Clin Chem 2000;46:650-657.

13. Wu TT, Yuan A, Chen CY, et al. Cardiac troponin I levels are a risk factor for mortality and multiple organ failure in noncardiac critically ill patients and have an additive effect to the APACHE II score in outcome prediction. Shock 2004;22:95101.

14. van Bockel EAP, Tulleken JE, Kobold ACM, et al. Cardiac troponin I release and cytokine response during experimental human endotoxaemia. Intensive Care Med 2003;29: $1598-1600$.

15. Alcalai R, Planer D, Culhaoglu A, et al. Acute coronary syndrome vs nonspecific troponin elevation-Clinical predictors and survival analysis. Arch Intern Med 2007;167:276-281.

16. Wu AHB. Increased troponin in patients with sepsis and septic shock: Myocardial necrosis or reversible myocardial depression? Intensive Care Med 2001;27:959-961.

17. Babuin L, Vasile VC, Perez JAR, et al. Elevated cardiac troponin is an independent risk factor for short- and long-term mortality in medical intensive care unit patients. Crit Care Med 2008;36:759-765.

18. Turner A, Tsamitros M, Bellomo R. Myocardial cell injury in septic shock. Crit Care Med 1999;27:1775-1780.

19. Langhorn R, Willesen J, Tarnow I, Kjelgaard-Hansen M. Evaluation of a high sensitivity assay for measurement of canine and feline cardiac troponin-I. Vet Clin Pathol 2013; in press.

20. DeFrancesco T, Atkins C, Keene B, et al. Prospective clinical evaluation of serum cardiac troponin $\mathrm{T}$ in dogs admitted to a veterinary teaching hospital. J Vet Intern Med 2002;16: 553-557.

21. Kjelgaard-Hansen M, Jensen A, Kristensen A. Evaluation of a commercially available human C-reactive protein (CRP) turbidometric immunoassay for determination of canine serum CRP concentration. Vet Clin Pathol 2003;32:81-87.

22. Hosmer D, Hosmer T, leCessie S, Lemeshow S. A comparison of goodness-of-fit tests for the logistic regression model. Stat Med 1997;16:965-980. 
23. Schober KE, Cornand C, Kirbach B, et al. Serum cardiac troponin $\mathrm{I}$ and cardiac troponin $\mathrm{T}$ concentrations in dogs with gastric dilatation-volvulus. J Am Vet Med Assoc 2002;221:381-388.

24. Shaw SP, Rozanski EA, Rush JE. Cardiac troponins I and T in dogs with pericardial effusion. J Vet Intern Med 2004;18:322-324.

25. Linklater AKJ, Lichtenberger MK, Thamm DH, et al. Serum concentrations of cardiac troponin I and cardiac troponin $\mathrm{T}$ in dogs with class IV congestive heart failure due to mitral valve disease. J Vet Emerg Crit Care 2007;17:243-249.

26. Labugger R, Organ L, Collier C, et al. Extensive troponin $\mathrm{I}$ and $\mathrm{T}$ modification detected in serum from patients with acute myocardial infarction. Circulation 2000;102:1221-1226.

27. Lim W, Whitlock R, Khera V, et al. Etiology of troponin elevation in critically ill patients. J Crit Care 2010;25:322-328.

28. Ilva TJ, Eskola MJ, Nikus KC, et al. The etiology and prognostic significance of cardiac troponin I elevation in unselected emergency department patients. J Emerg Med 2010;38:1-5.

29. Hayes G, Mathews $\mathrm{K}$, Kruth $\mathrm{S}$, et al. Illness severity scores in veterinary medicine: What can we learn? J Vet Intern Med 2010;24:457-466.

30. Rohrbach B, Buchanan B, Drake J, et al. Use of a multivariable model to estimate the probability of discharge in hospitalized foals that are 7 days of age or less. J Am Vet Med Assoc 2006;228:1748-1756.

31. Charpentier J, Luyt CE, Fulla Y, et al. Brain natriuretic peptide: A marker of myocardial dysfunction and prognosis during severe sepsis. Crit Care Med 2004;32:660-665.

32. Martin RS, Kincaid EH, Russell HM, et al. Selective management of cardiovascular dysfunction in posttraumatic SIRS and sepsis. Shock 2005;23:202-208.
33. Parker MM. Pathophysiology of cardiovascular dysfunction in septic shock. New Horiz Sci Pract Acute Med 1998;6:130-138.

34. Altmann DR, Korte W, Maeder MT, et al. Elevated cardiac troponin I in sepsis and septic shock: No evidence for thrombus associated myocardial necrosis. PLoS One 2010;5: e9017.

35. Beranek JT. Cardiomyocyte apoptosis contributes to the pathology of the septic shock heart. Intensive Care Med 2002;28:218.

36. Bowen FW, Hattori T, Narula N, et al. Reappearance of myocytes in ovine infarcts produced by six hours of complete ischemia followed by reperfusion. Ann Thorac Surg 2001;71:1845-1855.

37. Brett J, Gerlach H, Nawroth P, et al. Tumor necrosis factor cachectin increases permeability of endothelial-cell monolayers by a mechanism involving regulatory G-proteins. J Exp Med 1989;169:1977-1991.

38. Piper HM, Schwartz P, Spahr R, et al. Early enzymerelease from myocardial-cells is not due to irreversible celldamage. J Mol Cell Cardiol 1984;16:385-388.

39. Kumar A, Thota V, Dee L, et al. Tumor necrosis factor alpha and interleukin 1 beta are responsible for in vitro myocardial cell depression induced by human septic shock serum. J Exp Med 1996;183:949-958.

40. Bigalke B, Schwimmbeck PL, Haas CS, Lindemann S. Effect of interleukin-15 on the course of myocarditis in coxsackievirus B3-infected BALB/c mice. Can J Cardiol 2009;25: 248-254. 